\title{
IVORY'S THEOREM IN HYPERBOLIC SPACES
}

\author{
H. STACHEL AND J. WALLNER
}

\begin{abstract}
According to the planar version of Ivory's Theorem the family of confocal conics has the property that in each quadrangle formed by two pairs of conics the diagonals are of equal length. It turned out that this theorem is closely related to self-adjoint affine transformations. This point of view is capable of generalization to hyperbolic and other spaces.
\end{abstract}

\section{INTRODUCTION}

The planar Euclidean version of Ivory's Theorem states that the two diagonals $X_{1} X_{2}^{\prime}$ and $X_{2} X_{1}^{\prime}$ of any curvilinear quadrangle formed by four confocal conics have the same length (this is illustrated in Fig. 1). Another version of this theorem is the following: Assume that $k, k^{\prime}$ are confocal ellipses or confocal hyperbolas (two conics of the same type). Then there is an affine transformation $\alpha$ with $\alpha(k)=k^{\prime}$ and such that whenever a conic of the other type, but confocal with $k$ and $k^{\prime}$ intersects the conic $k$ in the point $X_{1}$, it will intersect $k^{\prime}$ in the point $\alpha\left(X_{1}\right)$, and both intersections are orthogonal. Now Ivory's Theorem states equal distances

$$
\overline{\alpha\left(X_{1}\right) X_{2}}=\overline{X_{1} \alpha\left(X_{2}\right)} \text { for all } X_{1}, X_{2} \in k \text {. }
$$

This statement holds also for singular $\alpha$ when $k^{\prime}=\alpha(k)$ degenerates into a set of points located on an axis of symmetry of $k$.

In $1809 \mathrm{~J}$. Ivory proved the three-dimensional version of this theorem by straightforward calculation and by using an appropriate parametrization ([7], see also $[5,11,4,1])$.

This theorem holds in the $n$-dimensional Euclidean space $(n>1$, see e.g. [9]). It has been shown by [8] that it is also true in the pseudo-Euclidean (Minkowski) plane. The aim of this paper is to prove Ivory's Theorem in hyperbolic spaces $\mathbb{H}^{n}$, and indeed in a more general class of pseudo-Riemannian spaces of constant curvature.

\section{Definitions AND BASIC RESUlts}

2.1. Scalar products. We consider the real $n$-dimensional projective space $\mathbb{P}^{n}$, which is the set of points $X=\mathbf{x} \mathbb{R}$ with $\mathbf{x} \in \mathbb{R}^{n+1} \backslash\{0\}$. We assume that $\mathbb{R}^{n+1}$ is endowed with a scalar product, i.e., a nondegenerate symmetric bilinear form

1991 Mathematics Subject Classification. 51M10, 15A21.

Key words and phrases. hyperbolic geometry, Ivory's Theorem, confocal quadrics. 


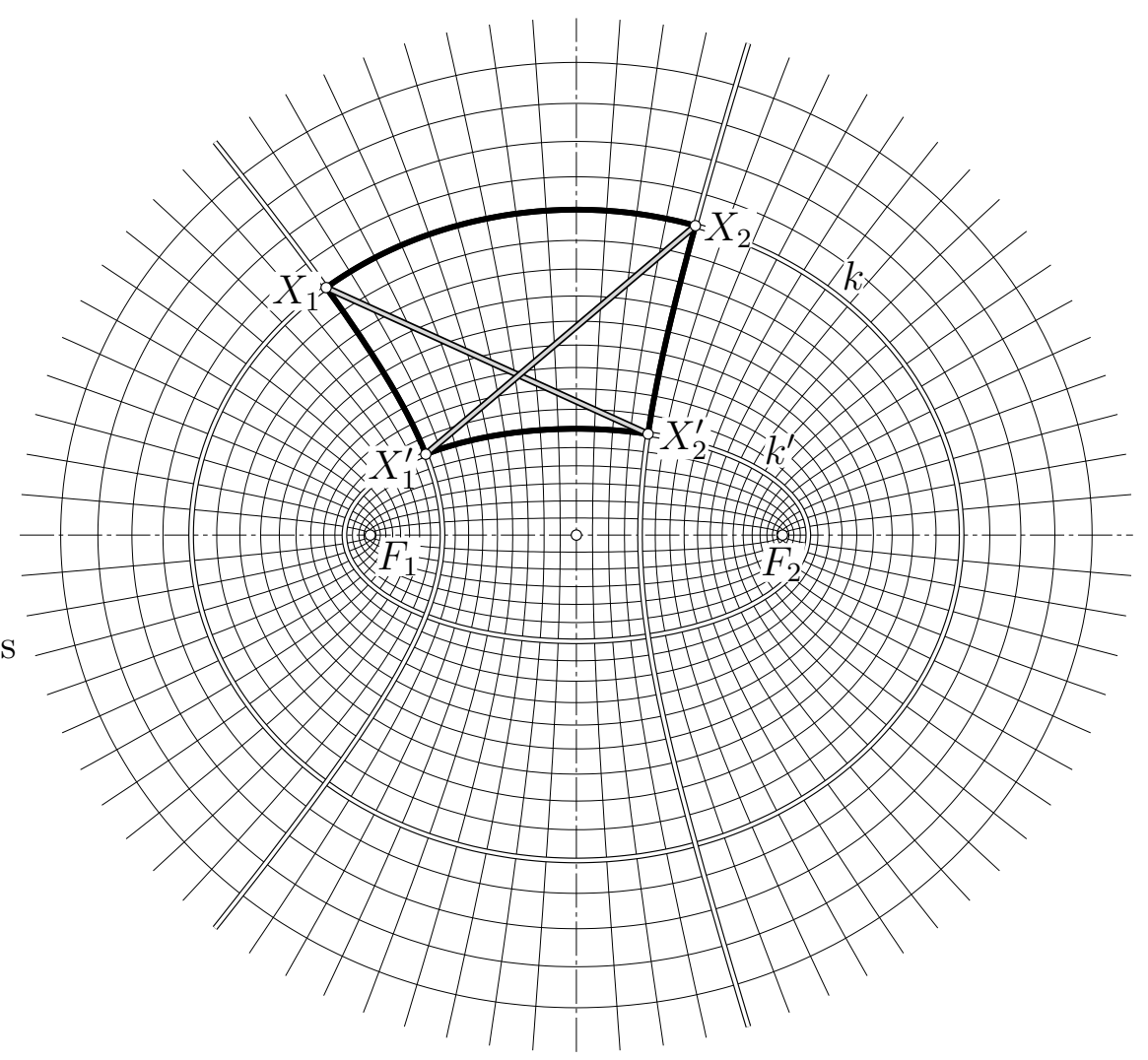

Figure 1. IvORY's Theorem in the Euclidean plane $\mathbb{E}^{2}$

$\langle$,$\rangle . If the scalar product has signature (-+\cdots+)$, then the $n$-dimensional hyperbolic space is given by the subset

$$
\mathbb{H}^{n}=\{\mathbf{x} \mathbb{R} \mid\langle\mathbf{x}, \mathbf{x}\rangle<0\}
$$

of $\mathbb{P}^{n}$. The hyperbolic distance $d_{h}(\mathbf{x}, \mathbf{y})$ of points of $\mathbb{H}^{n}$ is given by

$$
\cosh d_{h}(\mathbf{x}, \mathbf{y})=\left|\frac{\langle\mathbf{x}, \mathbf{y}\rangle}{\sqrt{\langle\mathbf{x}, \mathbf{x}\rangle\langle\mathbf{y}, \mathbf{y}\rangle}}\right| .
$$

The case of signature $(+\cdots+)$ leads to the elliptic metric $d_{e}$ in $\mathbb{P}^{n}$ defined by

$$
\cos d_{e}(\mathbf{x}, \mathbf{y})=\left|\frac{\langle\mathbf{x}, \mathbf{y}\rangle}{\sqrt{\langle\mathbf{x}, \mathbf{x}\rangle\langle\mathbf{y}, \mathbf{y}\rangle}}\right| .
$$

Remark. Standard spherical geometry is a twofold covering of the elliptic $n$-space. We may identify $\mathbb{H}^{n}$ with one of the two components of the set $\{\mathbf{x} \mid\langle\mathbf{x}, \mathbf{x}\rangle=-1\}$. This embedding induces a Riemannian metric in $\mathbb{H}^{n}$, where distances of points are given by (1). For general information on hyperbolic spaces see [2]. 
The signatures $(+-\cdots-)$ and $(-\cdots-)$ likewise generate hyperbolic and elliptic geometries, with the obivous modifications in the definition of the metric. Signatures of the form $(++-- \pm \cdots \pm)$ do not lead to Riemannian manifolds, but we may still consider expressions of the form

$$
\delta(X, Y)=\delta(\mathbf{x}, \mathbf{y})=\frac{\langle\mathbf{x}, \mathbf{y}\rangle}{\sqrt{|\langle\mathbf{x}, \mathbf{x}\rangle\langle\mathbf{y}, \mathbf{y}\rangle|}}
$$

which serve as a substitute for the metric, and which are meaningful for all points $X=\mathbf{x} \mathbb{R}, Y=\mathbf{y} \mathbb{R} \in \mathbb{P}^{n}$ with $\langle\mathbf{x}, \mathbf{x}\rangle,\langle\mathbf{y}, \mathbf{y}\rangle \neq 0$. Points $\mathbf{x} \mathbb{R}$ with $\langle\mathbf{x}, \mathbf{x}\rangle=0$ are called absolute.

The hyperplanes in $\mathbb{P}^{n}$ are the zero sets of linear forms $\mathbf{a}^{*} \in \mathbb{R}^{(n+1) *}$. We may represent $\mathbf{a}^{*}$ by its gradient vector $\mathbf{v}$ such that $\mathbf{a}^{*}(\mathbf{x})=\langle\mathbf{v}, \mathbf{x}\rangle$. The two hyperplanes with gradient vectors $\mathbf{v}, \mathbf{w}$, resp., are called orthogonal if $\langle\mathbf{v}, \mathbf{w}\rangle=0$. Self-orthogonal hyperplanes are called absolute.

Recall that endomorphisms $l$ and $l^{*}$ are called adjoint, if $\langle\mathbf{x}, l(\mathbf{y})\rangle=\left\langle l^{*}(\mathbf{x}), \mathbf{y}\right\rangle$ for all $\mathbf{x}, \mathbf{y}$. When we write bilinear forms as $\sigma(\mathbf{x}, \mathbf{y})=\langle\mathbf{x}, l(\mathbf{y})\rangle$ with a linear endomorphism $l$, then $\sigma$ is symmetric if and only if $l$ is selfadjoint.

Definition 1. A (nondegenerate) quadric $\Phi$ is the zero set of a (nondegenerate) symmetric bilinear form $\sigma(\mathbf{x}, \mathbf{y})=\langle\mathbf{x}, l(\mathbf{y})\rangle$, with a selfadjoint (nonsingular) linear endomorphism $l$.

$l=$ id corresponds to the absolute quadric $\Omega$, the set of absolute points.

2.2. Selfadjoint endomorphisms. We are going to describe simultaneous normal forms of the symmetric bilinear form $\langle$,$\rangle and the selfadjoint linear mappings$ $l$ - this means the choice of a coordinate system such that both have simple coordinate matrices. We use the notation $I_{k}$ for the $k \times k$ unit matrix, and

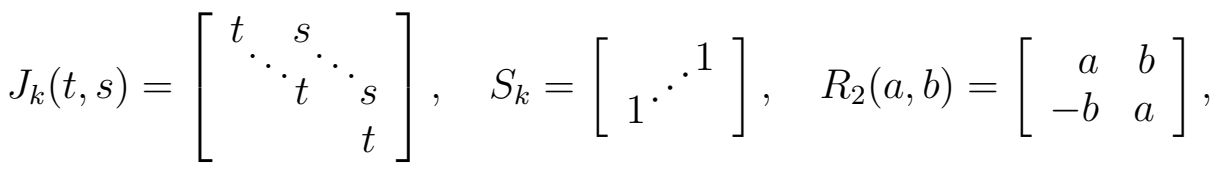

$$
\begin{aligned}
& R_{2 k}(a, b, s)=\left[\begin{array}{cccc}
R_{2}(a, b) & s I_{2} & & \\
\ddots & & \ddots & \\
& R_{2}(a, b) & s I_{2} \\
& & R_{2}(a, b)
\end{array}\right] .
\end{aligned}
$$

The lower right index indicates the size of the matrix.

Theorem 1. For any selfadjoint $l$, there are coordinates in $\mathbb{R}^{n+1}$ such that $\langle\mathbf{x}, \mathbf{y}\rangle=\mathbf{x}^{T} H \mathbf{y}, l(\mathbf{x})=A \cdot \mathbf{x}$, and the matrices $A, H$ have the form

(4) $\quad A=\operatorname{diag}\left(J_{r_{0}}\left(t_{0}, 1\right), \ldots, J_{r_{k-1}}\left(t_{k-1}, 1\right), R_{2 r_{k}}\left(a_{k}, b_{k}, 1\right), \ldots, R_{2 r_{s}}\left(a_{s}, b_{s}, 1\right)\right)$,

(5) $H=\operatorname{diag}\left(\epsilon_{0} S_{r_{0}}, \ldots, \epsilon_{k-1} S_{r_{k-1}}, \epsilon_{k} S_{2 r_{k}}, \ldots, \epsilon_{s} S_{2 r_{s}}\right)$,

with $\epsilon_{i}= \pm 1$ and $t_{i}, a_{i}, b_{i} \in \mathbb{R}, b_{i} \neq 0$.

Proof: See [6], Th. 5.3. 
Corollary 1. In case of a definite scalar product $\langle$,$\rangle , the normal form of Th. 1$ is the following:

$$
A=\operatorname{diag}\left(t_{0}, \ldots, t_{n}\right), \quad H= \pm I_{n+1} .
$$

In case of a hyperbolic scalar product with signature $\epsilon(-+\cdots+)$ the normal form of Th. 1 is one of the following:

(i) $\quad A=\operatorname{diag}\left(t_{0}, \ldots, t_{n}\right)$

$$
H=\epsilon \operatorname{diag}\left(-1, I_{n}\right),
$$

(ii) $\quad A=\operatorname{diag}\left(J_{2}\left(t_{0}, 1\right), t_{2}, \ldots, t_{n}\right) \quad H=\epsilon \operatorname{diag}\left(\epsilon_{0} S_{2}, I_{n-1}\right) \quad$ with $\epsilon_{0}= \pm 1$,

(iii) $A=\operatorname{diag}\left(R_{2}(a, b), t_{2}, \ldots, t_{n}\right) \quad H=\epsilon \operatorname{diag}\left(\epsilon_{0} S_{2}, I_{n-1}\right) \quad$ with $\epsilon_{0}= \pm 1$,

(iv) $\quad A=\operatorname{diag}\left(J_{3}\left(t_{0}, 1\right), t_{3}, \ldots, t_{n}\right) \quad H=\epsilon \operatorname{diag}\left(S_{3}, I_{n-2}\right)$.

Proof: We consider the hyperbolic case first. The signature of each matrix $\epsilon S_{r}$ is given by $\epsilon(+-+-\cdots)$, and by summing up we easily compute the signature of the block matrix (5). This signature must equal the signature of $\langle$,$\rangle . Thus$ only one of the $S_{r}$ 's can have size greater than one, and this size must be less than four. The result follows immediately from Th. 1 .

The elliptic case is similar - besides, this is the spectral theorem for selfadjoint endomorphisms in the presence of a definite scalar product.

2.3. Square roots. Later we will need that certain endomorphisms have square roots of a certain form, so we collect some lemmas here. We will extend the definition of $J_{k}(t, s)$ to matrices with entries in a ring $R$ in the obvious way. First we define a relation between formal power series with coefficients in a commutative ring $R$ and matrices in $R^{k \times k}(k>0)$ as follows:

$$
a(x) \sim A \Longleftrightarrow a(x)=\sum a_{i} x^{i}, A=\left[\begin{array}{cccc}
a_{0} & a_{1} & \ldots & a_{k-1} \\
& a_{0} & \ldots & a_{k-2} \\
& & \ddots & \vdots \\
& & & a_{0}
\end{array}\right] .
$$

It is obvious that

$$
A, B \in R^{k \times k}, a(x) \sim A, b(x) \sim B \Longrightarrow a(x) b(x) \sim A B .
$$

Lemma 1. Assume that $R$ is a $\mathbb{Q}$-algebra (i.e., a commutative ring which contains $\mathbb{Q}$ as a subring). Assume further that $t \in R$ has a square root $\sqrt{t}$ as well as an inverse $t^{-1}$. Consider the upper triangular matrix $A \in R^{k \times k}$ which according to $(6)$ is related to the power series

$$
a(x)=(t+s x)^{1 / 2}=\sum_{i \geq 0}\left(\begin{array}{c}
1 / 2 \\
i
\end{array}\right)\left(s t^{-1}\right)^{i} \sqrt{t} x^{i} \in R[[x]] .
$$

Then $A^{2}$ equals the Jordan block $J_{k}(t, s)$.

Proof: Clearly, the matrix $J_{k}(t, s)$ is related to the power series $t+s x$, and by (7) a square root is found as the matrix related to $(t+s x)^{1 / 2}=\sqrt{t}\left(1+s t^{-1}\right)^{1 / 2}=$ $\sqrt{t} \sum_{i \geq 0}\left(\begin{array}{c}1 / 2 \\ i\end{array}\right)\left(s t^{-1}\right)^{i} x^{i}$. 
Lemma 2. A Jordan block $J_{k}(t, s) \in \mathbb{R}^{k \times k}$ with $t>0$ has a square root of the form (6) with $a_{i}=\left(\begin{array}{c}1 / 2 \\ i\end{array}\right)(s / t)^{i} \sqrt{t}$. All matrices $R_{2 k}(a, b, s) \in \mathbb{R}^{2 k \times 2 k}$ with $b \neq 0$ have square roots of the following forms:

$$
\begin{aligned}
& \sqrt{R_{2}(a, b)}=R_{2}(b / 2 \zeta, \zeta) \text {, where } 4 \zeta^{4}+4 a \zeta^{2}-b^{2}=0,
\end{aligned}
$$

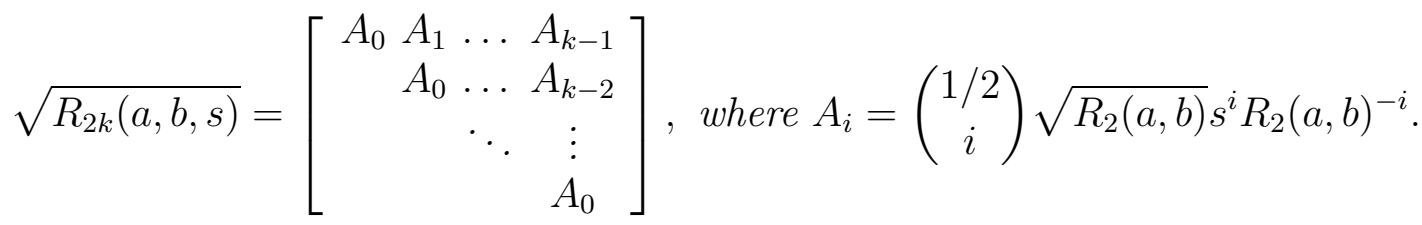

Proof: The statement about $J_{k}(t, s)$ follows directly from Lemma 1 if we let $R=$ $\mathbb{R}$. The statement about $R_{2}(a, b)$ is easily verified. Observe that the quadratic equation $4 \xi^{2}+4 a \xi-b^{2}=0$ has the solutions $\xi=\frac{1}{2}\left(-a \pm \sqrt{a^{2}+b^{2}}\right)$, at least one of which is positive because $b \neq 0$. This shows that $\zeta$ exists and is nonzero.

Now let $R$ equal the subring of the matrix ring $\mathbb{R}^{2 \times 2}$ which is generated by the matrix $\sqrt{R_{2}(a, b)}$. Note that the matrix ring $R^{k \times k}$ is embedded into the matrix ring $\mathbb{R}^{2 k \times 2 k}$, and that $J_{k}\left(R_{2}(a, b), s I_{2}\right)=R_{2 k}(a, b, s)$. With these identifications, the statement about $\sqrt{R_{2 k}(a, b, s)}$ follows directly from Lemma 1.

Corollary 2. Selfadjoint linear endomorphisms l in $\mathbb{R}^{n+1}$ whose real eigenvalues are positive possess selfadjoint square roots.

Proof: We use the coordinate matrices $A$ of $l$ and $H$ of $\langle$,$\rangle given by Th. 1$ which have a common block diagonal structure. It is sufficient to consider each block $A_{k}$ and $H_{k}= \pm S_{k}$ separately. Square roots $\sqrt{A}_{k}$ are given by Lemma 2 . As ${\sqrt{A_{k}}}^{T}\left( \pm S_{k}\right)=\left( \pm S_{k}\right) \sqrt{A_{k}}$ in each case, the mapping $\sqrt{l}$ constructed in this way is selfadjoint.

Corollary 3. Both the matrices $J_{k}(t, s)$ for $t>0$ and $R_{2 k}(a, b, s)$ with $a^{2}+b^{2}>0$ have square roots which depend smoothly on $t, s$ and on $a, b, s$, respectively. The derivatives commute with the matrices.

Proof: The result concerning smoothness follows from the explicit formula for the square roots given by Lemma 2 - we use the fact that $\zeta$ equals $\left(\frac{1}{2}\left(-a+\left(a^{2}+\right.\right.\right.$ $\left.\left.\left.b^{2}\right)^{\frac{1}{2}}\right)\right)^{\frac{1}{2}}$. We observe that derivatives of matrices of the form (6) are again of that form. Now commutativity follows from (7), as multiplication of power series is commutative.

2.4. Dual quadrics. Points $X=\mathbf{x} \mathbb{R}, Y=\mathbf{y} \mathbb{R}$ are called conjugate with respect to a nondegenerate quadric $\Phi$ or its defining bilinear form $\sigma$, if $\sigma(\mathbf{x}, \mathbf{y})=$ $\langle\mathbf{x}, l(\mathbf{y})\rangle=0$.

The set of points conjugate to $X$ is the hyperplane with gradients $\lambda l(\mathbf{x})$. If points $X, Y$ are contained in a quadric $\Phi$, and they are conjugate with respect to $\Phi$, then the line $X \vee Y$ is contained in $\Phi$. Conversely, any two points of a subspace contained in $\Phi$ are conjugate. 
The tangent hyperplane of $\Phi$ in $\mathbf{x}$ has gradients $\lambda l(\mathbf{x})$. Conversely, a hyperplane with gradient $\mathbf{v}$ is tangent to $\Phi$ if and only if $\widehat{\sigma}(\mathbf{v}, \mathbf{v})=\sigma\left(l^{-1}(\mathbf{v}), l^{-1}(\mathbf{v})\right\rangle=$ $\left\langle l^{-1}(\mathbf{v}), \mathbf{v}\right\rangle=\left\langle\mathbf{v}, l^{-1}(\mathbf{v})\right\rangle=0$. If $\mathbf{a}^{*}, \mathbf{b}^{*}$ have respective gradient vectors $\mathbf{v}, \mathbf{w}$, then we consider the bilinear form

$$
\sigma^{*}\left(\mathbf{a}^{*}, \mathbf{b}^{*}\right)=\widehat{\sigma}(\mathbf{v}, \mathbf{w})=\left\langle\mathbf{v}, l^{-1}(\mathbf{w})\right\rangle .
$$

Definition 2. The quadric $\widehat{\Phi}$ in the dual space defined by (9) is called the dual of the original quadric $\Phi$ defined by $\sigma$.

If we use coordinates with respect to some basis, we assume linear forms to be coordinatized with respect to the corresponding dual basis. If $\langle\mathbf{x}, \mathbf{y}\rangle=\mathbf{x}^{T} H \mathbf{y}$, then a linear form $\mathbf{a}^{*}$ and its gradient $\mathbf{v}$ are connected via $\mathbf{a}^{*}=H \mathbf{v}$. If $\sigma(\mathbf{x}, \mathbf{y})=$ $\mathbf{x}^{T} H A \mathbf{y}$, we have $\widehat{\sigma}(\mathbf{v}, \mathbf{w})=\mathbf{v}^{T} H A^{-1} \mathbf{w}$, and $\sigma^{*}\left(\mathbf{a}^{*}, \mathbf{b}^{*}\right)=\mathbf{a}^{* T}(H A)^{-1} \mathbf{b}^{*}$.

The dual quadric $\widehat{\Phi}$ may be seen as a set of hyperplanes (the zero sets of the corresponding linear forms). It is the image of $\Phi$ under the mapping " $X \mapsto$ hyperplane of points conjugate to $X$ ".

We have excluded singular quadrics and singular forms from this discussion about dual quadrics - they do not possess duals in the sense of Def. 2. There are however singular dual quadrics defined by singular symmetric bilinear forms in $\mathbb{R}^{(n+1) *}$.

We compute the image $\Phi_{1}=k\left(\Phi_{0}\right)$ of a quadric $\Phi_{0}$ under a nonsingular endomorphism $k$ : We have $\mathbf{x} \in \Phi_{0} \Longleftrightarrow k(\mathbf{x}) \in \Phi_{1}$. Thus if $\Phi_{0}$ is defined by the bilinear form $\sigma_{0}(\mathbf{x}, \mathbf{y})=\langle\mathbf{x}, l(\mathbf{y})\rangle$, then $\Phi_{1}$ is defined by $\sigma_{1}(\mathbf{x}, \mathbf{y})=\left\langle k^{-1}(\mathbf{x}), l k^{-1}(\mathbf{y})\right\rangle=$ $\left\langle\mathbf{x},\left(k^{-1}\right)^{*} l k^{-1}(\mathbf{y})\right\rangle$. If we represent linear forms by their gradients, the respective duals of $\Phi_{0}$ and $\Phi_{1}$ are defined by bilinear forms $\left\langle\mathbf{v}, l^{-1}(\mathbf{w})\right\rangle$ and $\left\langle\mathbf{v}, k l^{-1} k^{*}(\mathbf{w})\right\rangle$.

The fact that the inverse of $k$ does not appear in the form which defines $\widehat{\Phi}_{1}$ allows to extend these computations by the following definition:

Definition 3. If $k$ is a linear endomorphism and the quadric $\Phi$ is given by the endomorphism $l$, then we define the dual $k$-image of $\Phi$ to have the equation

$$
\widehat{\sigma}(\mathbf{v}, \mathbf{v})=0, \text { with } \widehat{\sigma}(\mathbf{v}, \mathbf{w})=\left\langle\mathbf{v}, k l^{-1} k^{*}(\mathbf{w})\right\rangle .
$$

$\widehat{\sigma}$ is understood to apply to gradients.

It so appears that singular linear mappings, when applied to nonsingular quadrics, still define meaningful dual image quadrics.

2.5. Families of confocal forms and quadrics. Assume that the quadrics $\Phi_{0}$ and $\Phi_{1}$ are defined by symmetric bilinear forms $\sigma_{0}$ and $\sigma_{1}$, or selfadjoint endomorphisms $l_{0}$ and $l_{1}$, respectively, and that their respective duals $\widehat{\Phi}_{0}, \widehat{\Phi}_{1}$ are represented by the symmetric bilinear forms $\widehat{\sigma}_{0}$ and $\widehat{\sigma}_{1}$ according to Def. 2 .

In coordinates, we let $l_{i}(\mathbf{x})=A_{i} \mathbf{x}$ and $\langle\mathbf{x}, \mathbf{y}\rangle=\mathbf{x}^{T} H \mathbf{y}$. Then $\sigma_{i}(\mathbf{x}, \mathbf{y})=\mathbf{x}^{T} Q_{i} \mathbf{y}$ with $Q_{i}=H A_{i}$, and $\sigma_{i}^{*}\left(\mathbf{a}^{*}, \mathbf{b}^{*}\right)=\mathbf{a}^{* T} Q_{i}^{-1} \mathbf{b}^{*}$. 
Definition 4. We use the notations defined immediately above. $\Phi_{0}$ and $\Phi_{1}$ are said to be confocal (or homofocal), if one of the following equivalent conditions holds true:

(i) the bilinear forms $\widehat{\sigma}_{0}, \widehat{\sigma}_{1}, \widehat{\langle,\rangle}=\langle$,$\rangle are linearly dependent,$

(ii) the linear endomorphisms $l_{0}^{-1}, l_{1}^{-1}$, id are linearly dependent,

(iii) the coordinate matrices $Q_{1}^{-1}, Q_{2}^{-1}$, and $H^{-1}$ are linearly dependent.

The family of quadrics $\Phi$ confocal to $\Phi_{0}$ is defined by endomorphisms $l$ which satisfy $l^{-1}=\lambda l_{0}^{-1}+\mu \mathrm{id},(\lambda, \mu) \in \mathbb{R}^{2}, \lambda \neq 0$.

Hence the dual quadrics $\widehat{\Phi}$ together with the dual $\widehat{\Omega}$ of the absolute quadric form a linear system of algebraic hypersurfaces in the dual projective space. Any absolute tangent hyperplane of $\Phi$ is also tangent to $\Phi_{0}$, and vice versa.

Remark. This definition extends the usual definition of confocality in Euclidean geometry, which fits into this framework after introducing homogeneous coordinates and defining a singular symmetric bilinear form $\widehat{\langle,\rangle}$, cf. $[5,11,4,3]$.

In the Euclidean plane, confocal conics (see Fig. 1) may be of different type, i.e., one of them an ellipse, and another a hyperbola. The obvious fact that there is no continuous transition from an ellipse to a hyperbola is the motivation for the following definition.

Definition 5. Within the family of confocal bilinear forms spanned by $l_{0}$, the connected components of $\left\{(\lambda, \mu) \mid \lambda\left(l_{0}^{-1}+\mu \mathrm{id}\right)\right.$ nonsingular $\}$ correspond to quadrics of different types.

Lemma 3. In the $n$-dimensional elliptic or hyperbolic space $(n>1)$ all confocal families possess at least two types of quadrics.

Proof: By Def. 5 it is sufficient to show that $l_{0}^{-1}$ has an eigenvalue. As $l_{0}^{-1}$ is selfadjoint, we apply Cor. 1 and the fact that $n+1 \geq 3$.

The following is well known:

Lemma 4. If confocal quadrics $\Phi_{0}$ and $\Phi_{\lambda}$ intersect, they do so orthogonally.

Proof: Assume that $g_{0}$ and $g_{\lambda}$ are endomorphisms which define confocal quadrics, such that $g_{\lambda}^{-1}=g_{0}^{-1}+\lambda$ id $(\lambda \neq 0)$, and that $\mathbf{v}=g_{0}(\mathbf{x})$ and $\mathbf{w}=g_{\lambda}(\mathbf{x})$ are gradient vectors of tangent hyperplanes in $\mathbf{x} \in \Phi_{0} \cap \Phi_{\lambda}$.

From the relation $0=\left\langle\mathbf{x}, g_{0}(\mathbf{x})\right\rangle=\langle\mathbf{x}, \mathbf{v}\rangle=\left\langle g_{\lambda}^{-1}(\mathbf{w}), \mathbf{v}\right\rangle$ and the analogous relation $0=\left\langle g_{0}^{-1}(\mathbf{w}), \mathbf{v}\right\rangle$ we get $0=\langle\mathbf{v}, \mathbf{w}\rangle$ by linear combination.

\section{The IVORY PROPERTY AND SELFADJOINT ENDOMORPHISMS}

3.1. Ivory maps are linear. We show that Ivory's Theorem for two quadrics $\Phi_{0}, \Phi_{1}$ is always related to the existence of a selfadjoint linear endomorphism $l$ with $\Phi_{1}=l\left(\Phi_{0}\right)$.

Lemma 5. Assume that $\Phi$ is a quadric, possibly singular but not contained in a hyperplane, and that there is a mapping $\mathbf{x} \mapsto \mathbf{x}^{\prime}$ such that

$$
\left\langle\mathbf{x}_{1}^{\prime}, \mathbf{x}_{2}\right\rangle=\left\langle\mathbf{x}_{1}, \mathbf{x}_{2}^{\prime}\right\rangle \text { for all } \mathbf{x}_{1}, \mathbf{x}_{2} \in \Phi,
$$


then there is a selfadjoint linear endomorphism $l$ of $\mathbb{R}^{n+1}$ such that $\mathbf{x}^{\prime}=l(\mathbf{x})$ for all $\mathrm{x} \in \Phi$.

Proof: Choose linearly independent vectors $\mathbf{x}_{0}, \ldots, \mathbf{x}_{n} \in \Phi$ and define a linear endomorphism $l$ by $l\left(\mathbf{x}_{i}\right)=\mathbf{x}_{i}^{\prime}(i=0, \ldots, n)$. By our assumption, we have

$$
\left\langle\mathbf{x}_{i}, l^{*}(\mathbf{y})\right\rangle=\left\langle l\left(\mathbf{x}_{i}\right), \mathbf{y}\right\rangle=\left\langle\mathbf{x}_{i}^{\prime}, \mathbf{y}\right\rangle=\left\langle\mathbf{x}_{i}, \mathbf{y}^{\prime}\right\rangle \quad(i=0, \ldots, n) .
$$

By linear independence of the vectors $\mathbf{x}_{i}$, we conclude that $\mathbf{y}^{\prime}=l^{*}(\mathbf{y})$. This holds for all $\mathbf{y}$, so we especially have $l^{*}\left(\mathbf{x}_{i}\right)=\mathbf{x}_{i}^{\prime}$, which shows that $l=l^{*}$.

\subsection{The quadrics with equation $\langle\mathbf{x}, \mathbf{x}\rangle-\langle l(\mathbf{x}), l(\mathbf{x})\rangle=0$.}

Lemma 6. If the linear endomorphism $l$ is selfadjoint, then the quadric

$$
\Phi_{0}: \sigma(\mathbf{x}, \mathbf{x}):=\langle\mathbf{x}, \mathbf{x}\rangle-\langle l(\mathbf{x}), l(\mathbf{x})\rangle=0
$$

together with its l-image $\Phi_{1}$ has the Ivory property

$$
\delta(l(\mathbf{x}), \mathbf{y})=\delta(\mathbf{x}, l(\mathbf{y})) \text { for all } \mathbf{x}, \mathbf{y} \in \Phi \text { with }\langle\mathbf{x}, \mathbf{x}\rangle,\langle\mathbf{y}, \mathbf{y}\rangle \neq 0 .
$$

The restriction of $l$ to any linear subspace contained in $\Phi_{0}$ is isometric in the sense of $\delta$.

Proof: We have to show that

$$
\frac{\langle\mathbf{x}, l(\mathbf{y})\rangle}{\sqrt{\langle\mathbf{x}, \mathbf{x}\rangle\langle l(\mathbf{y}), l(\mathbf{y})\rangle}}=\frac{\langle l(\mathbf{x}), \mathbf{y}\rangle}{\sqrt{\langle l(\mathbf{x}), l(\mathbf{x})\rangle\langle\mathbf{y}, \mathbf{y}\rangle}}
$$

The denominators of these fractions are equal due to (10), whereas the numerators are equal because $l$ is selfadjoint.

As to the second statement, the distance $\delta$ is preserved for $\mathbf{x}, \mathbf{y} \in \Phi_{0}$ if and only if $\langle\mathbf{x}, \mathbf{y}\rangle=\langle l(\mathbf{x}), l(\mathbf{y})\rangle$. This equation characterizes conjugacy with respect to $\Phi_{0}$, and it follows that the restriction of $l$ to any subspace contained in $\Phi_{0}$ is isometric.

Lemma 7. Assume that $l$ is selfadjoint and that the quadric $\Phi_{0}$ given by (10) is regular. Then $\Phi_{0}$ and $\Phi_{1}=l\left(\Phi_{0}\right)$ are confocal (We apply Def. 3 if necessary).

Proof: We rewrite the equation of $\Phi_{0}$ :

$$
\sigma_{0}(\mathbf{x}, \mathbf{y})=\langle\mathbf{x}, \mathbf{y}\rangle-\langle l(\mathbf{x}), l(\mathbf{y})\rangle=\left\langle\mathbf{x},\left(\mathrm{id}-l^{2}\right)(\mathbf{y})\right\rangle .
$$

Its dual is represented by

$$
\widehat{\sigma}_{0}(\mathbf{v}, \mathbf{w})=\left\langle\mathbf{v},\left(\mathrm{id}-l^{2}\right)^{-1}(\mathbf{w})\right\rangle .
$$

The dual $l$-image of $\Phi_{0}$ is, according to Def. 3, defined by

$$
\widehat{\sigma}_{1}(\mathbf{v}, \mathbf{w})=\left\langle\mathbf{v}, l\left(\mathrm{id}-l^{2}\right)^{-1} l(\mathbf{w})\right\rangle .
$$

We show that

$$
\widehat{\sigma}_{0}-\widehat{\sigma}_{1}=\langle,\rangle
$$

by using $l\left(\mathrm{id}-l^{2}\right)=\left(\mathrm{id}-l^{2}\right) l$ and thus verifying that $\left(\mathrm{id}-l^{2}\right)^{-1}-l\left(\mathrm{id}-l^{2}\right)^{-1} l=$ id. 


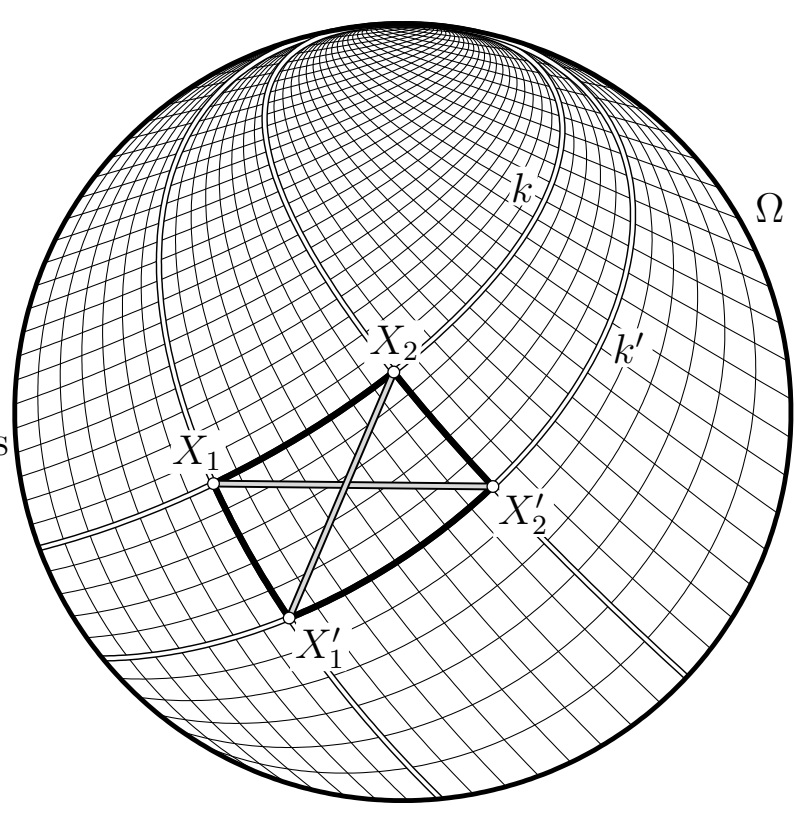

FiguRE 2. Ivory's Theorem in the hyperbolic plane $\mathbb{H}^{2}$ - case (iv) of Th. 1 which leads to conics without center and axis.

Lemma 8. If $l$ is selfadjoint, then in most cases the quadric $\Phi_{0}$ as defined by (10) is of the same type as $\Phi_{1}=l\left(\Phi_{0}\right)$ provided both are regular. Different types are only possible when the normal form of $l$ contains a block matrix $R_{2}(0, b)$ or $R_{2 k}(0, b, 1)$.

Proof: The quadric $\Phi_{i}$ is the zero set of $\sigma_{i}(\mathbf{x}, \mathbf{x})=\left\langle\mathbf{x}, g_{i}(\mathbf{x})\right\rangle$, with selfadjoint $g_{i}$ $(i=0,1)$. The proof of Lemma 7 shows that $\widehat{\sigma}_{0}-\widehat{\sigma}_{1}=\langle$, $\rangle$, i.e., $g_{0}^{-1}-\mathrm{id}=g_{1}^{-1}$. We ask if

$$
g_{\lambda}^{-1}=(1-\lambda) g_{0}^{-1}+\lambda g_{1}^{-1}=g_{0}^{-1}-\lambda \mathrm{id} \quad(0 \leq \lambda \leq 1)
$$

can be singular - if it is not for any $\lambda \in[0,1]$, then according to Def. $5, \Phi_{0}$ and $\Phi_{1}$ are of the same type (if it is, then the notation $g_{\lambda}^{-1}$ of course does not make sense).

By construction, we have $g_{0}^{-1}=\left(\mathrm{id}-l^{2}\right)^{-1}$. We use coordinates according to Th. 1. Because of the block matrix structure of (4) it is sufficient to assume that the coordinate matrix $A$ of $l$ equals either $J_{k}(t, 1)$ or $R_{2 k}(a, b, 1)$. In the first case, the coordinate matrix of $g_{\lambda}^{-1}$ reads

$$
\left(I_{k}-A^{2}\right)^{-1}-\lambda I_{k}=\left[\begin{array}{llr}
\left(1-t^{2}\right)^{-1}-\lambda & & * \\
& \ddots & \\
& & \left(1-t^{2}\right)^{-1}-\lambda
\end{array}\right] .
$$

As $\left(1-t^{2}\right)^{-1}$ does not assume values in $[0,1], g_{\lambda}^{-1}$ is nonsingular. In the second case $A=R_{2 k}(a, b, 1)$ with $b \neq 0$ we use the abbreviation $U=\left(I_{2}-R_{2}(a, b)^{2}\right)^{-1}$ 
and get

$$
\begin{gathered}
\left(I_{k}-A^{2}\right)^{-1}-\lambda I_{2 k}=\left[\begin{array}{lll}
U-\lambda I_{2} & & * \\
& \ddots & \\
& & U-\lambda I_{2}
\end{array}\right] \text {, with } \\
U=\frac{1}{\left(a^{2}+b^{2}+1\right)^{2}-4 a^{2}}\left[\begin{array}{cc}
1-a^{2}+b^{2} & 2 a b \\
-2 a b & 1-a^{2}+b^{2}
\end{array}\right]=R_{2}\left(a^{\prime}, b^{\prime}\right) .
\end{gathered}
$$

The eigenvalues of $U$ are $a^{\prime} \pm i b^{\prime}$. They are nonreal if $b^{\prime} \neq 0$, i.e., $a \neq 0$. So also in this case $g_{\lambda}^{-1}$ is nonsingular.

In the case $a=0$ the number $1 /\left(b^{2}+1\right)$ is the only eigenvalue of $U$, and it is contained in the interval $[0,1]$. Thus in this case there exists $\lambda \in(0,1)$ such that $g_{\lambda}$ is not defined.

\section{IVORY'S THEOREM}

4.1. A representation theorem. In previous lemmas we have enumerated properties of quadrics with special equations. Now we are going to show that this case is actually the general case we need for Ivory's Theorem.

Lemma 9. Consider two regular confocal quadrics $\Phi_{0}, \Phi_{1}$ which are of the same type. Then there is a selfadjoint endomorphism l such that $\Phi_{1}=l\left(\Phi_{0}\right)$ and the equation of $\Phi_{0}$ is given by $\langle\mathbf{x}, \mathbf{x}\rangle-\langle l(\mathbf{x}), l(\mathbf{x})\rangle=0$.

Proof: We assume that $\Phi_{i}$ is determined by the selfadjoint endomorphism $g_{i}$ $(i=0,1)$. By confocality, $g_{1}^{-1}=\lambda g_{0}^{-1}+\mu \mathrm{id}$. Without changing the quadrics we multiply $g_{0}$ and $g_{1}$ with real numbers such that

$$
g_{0}^{-1}-g_{1}^{-1}=\mathrm{id} .
$$

We first show that there exists $l$ such that $g_{0}=\mathrm{id}-l^{2}$, i.e., there exists a square root of id $-g_{0}$. We assume coordinate matrices $A$ and $H$ of $g_{0}$ and $\langle$,$\rangle as given$ by (4) and (5), respectively. By the block matrix structure of these matrices, it is sufficient to assume that $A=J_{k}(t, 1)$ or that $A=R_{2 k}(a, b, 1)$. In the first case, $g_{1}$ has the coordinate matrix

$$
\left(A^{-1}-I_{k}\right)^{-1}=\left[\begin{array}{lll}
\left(t^{-1}-1\right)^{-1} & & * \\
& \ddots & \\
& & \left(t^{-1}-1\right)^{-1}
\end{array}\right]
$$

By our assumption on the type, $t$ and $\left(t^{-1}-1\right)^{-1}$ have the same sign. A positive sign implies that $t \in(0,1)$ and a negative one implies that $t<0$. In both cases $1-t>0$, so id $-g_{0}$ has positive eigenvalues.

In the case that $A=R_{2 k}(a, b, 1)$ the matrix $I_{2 k}-A$ has the form $R_{2 k}(1-$ $a,-b,-1)$ with $b \neq 0$, and id $-g_{0}$ has no real eigenvalues. Thus in all cases we are able to apply Cor. 2 , and there is a selfadjoint endomorphism $l$ with $l^{2}=$ id $-g_{0}$. It remains to show that indeed $l\left(\Phi_{0}\right)=\Phi_{1}$. We have just shown 
that $\Phi_{0}$ has an equation of the form required by Lemma 7 , and by $(11), l\left(\Phi_{0}\right)$ is defined by an endomorphism $\bar{g}_{1}$ with the property that

$$
g_{0}^{-1}-\bar{g}_{1}^{-1}=\mathrm{id} .
$$

Our assumption (12) now shows $\bar{g}_{1}=g_{1}$ and the proof is complete.

Lemma 10. We use the notation of the proof of Lemma 9. There is $\delta>0$ such that id $-\lambda g_{0}$ has a square root which smoothly depends on $\lambda$, for $-\delta<\lambda<1+\delta$.

Proof: We again look at the proof of Lemma 9. In the case that $A=J_{k}(t, 1)$, we have $t \in(0,1)$ or $t<0$, which implies that there is $\delta$ such that $1-\lambda t>0$ for $\lambda \in(-\delta, 1+\delta)$. It follows that id $-\lambda g_{0}$ has a square root which according to Cor. 3 smoothly depends on $\lambda$.

In the case that $A=R_{2 k}(a, b, 1)$, the endomorphism id $-\lambda g_{0}$ has the coordinate matrix $R_{2 k}(1-\lambda a,-\lambda b,-\lambda)$. As there is no $\lambda$ such that $1-\lambda a=\lambda b=0$, we may apply Cor. 3 and conclude that there exists a square root of id $-\lambda g_{0}$ which smoothly depends on $\lambda$.

Remark. In a neighbourhood of $\lambda=0$ we can also apply the implicit function theorem to deduce that there is a selfajoint square root of id $-\lambda g_{0}$ which smoothly depends on $\lambda$.

4.2. Orthogonal trajectories of confocal families. We want to generalize the Euclidean theorem that corresponding points $X_{i}, X_{i}^{\prime}$ of confocal conics $k, k^{\prime}$ are located on another confocal conic which intersects the two original ones orthogonally (see Fig. 1).

Lemma 11. Suppose that $\Phi_{0}, \Phi_{1}, g_{0}, g_{1}, l$ are as in Lemma 9 and its proof. Then there is a smooth family $l_{\lambda}$ of endomorphisms with $l_{0}=\mathrm{id}$ and $l_{1}=l$, such that the quadric $\Phi_{\lambda}=l_{\lambda}\left(\Phi_{0}\right)$ is defined by the endomorphism $g_{\lambda}$ with

$$
g_{\lambda}^{-1}=g_{0}^{-1}-\lambda \mathrm{id}
$$

All quadrics $\Phi_{\lambda}$ are confocal with $\Phi_{0}$. They orthogonally intersect the path $l_{\lambda}(\mathbf{x}) \mathbb{R}$ of a point $\mathbf{x} \mathbb{R} \in \Phi_{0}$.

Proof: We have $g_{0}=\mathrm{id}-l^{2}$ and $g_{1}^{-1}=g_{0}^{-1}-\mathrm{id}$. We consider $\lambda g_{0}$ instead of $g_{0}$ and define $l_{\lambda}$ by

$$
\lambda g_{0}=\mathrm{id}-l_{\lambda}^{2} .
$$

By Lemma 10, $l_{\lambda}$ exists and depends smoothly on $\lambda$. We observe that $l_{\lambda}$ and $g_{0}$ commute, which for $\lambda=0$ is obvious and otherwise follows from $l_{\lambda} g_{0}=l_{\lambda} \lambda^{-1}$ (id$\left.l_{\lambda}^{2}\right)=\lambda^{-1}\left(\mathrm{id}-l_{\lambda}^{2}\right) l_{\lambda}=g_{0} l_{\lambda}$. Now we compute the endomorphism $g_{\lambda}^{-1}$ which defines the dual of $l_{\lambda}\left(\Phi_{0}\right)$ according to Def. 3:

$$
g_{\lambda}^{-1}=l_{\lambda} g_{0}^{-1} l_{\lambda}=l_{\lambda}^{2} g_{0}^{-1}=\left(\mathrm{id}-\lambda g_{0}\right) g_{0}^{-1}=g_{0}^{-1}-\lambda \mathrm{id} .
$$


So we have shown (13). Confocality of $\Phi_{0}$ and $l_{\lambda}\left(\Phi_{0}\right)$ follows from Lemma 7 by construction. We differentiate the relation $\lambda g_{0}=\mathrm{id}-l_{\lambda} l_{\lambda}$ and get

$$
g_{0}=-\left(\dot{l}_{\lambda} l_{\lambda}+l_{\lambda} \dot{l}_{\lambda}\right)=-2 \dot{l}_{\lambda} l_{\lambda} \Longrightarrow \dot{l}_{\lambda}=-\frac{1}{2} g_{0} l_{\lambda}^{-1}=-\frac{1}{2} g_{\lambda} l_{\lambda} .
$$

We have used $g_{\lambda}=g_{0} l_{\lambda}^{-2}$ and the fact that $l_{\lambda}$ and $\dot{l}_{\lambda}$ commute, which follows from Th. 1 and Cor. 3.

The tangent hyperplane of $\Phi_{\lambda}$ in $l_{\lambda}(\mathbf{x})$ has the gradient vector $g_{\lambda} l_{\lambda}(\mathbf{x})$. (14) implies that the tangent point $\dot{l}_{\lambda}(\mathbf{x}) \mathbb{R}$ is conjugate to this tangent hyperplane with respect to the absolute quadric $\Omega$.

Lemma 12. We use the notations of Lemma 11 and consider the quadrics $\Phi_{\lambda}$, defined by endomorphisms $g_{\lambda}$. If $\Psi$ is confocal with $\Phi_{0}$, but different from $\Phi_{0}$, and $\mathbf{x} \in \Phi_{0} \cap \Psi$, then also $l_{\lambda}(\mathbf{x}) \in \Psi$.

Proof: We have $\mathbf{x} \in \Phi_{0} \Longleftrightarrow\left\langle\mathbf{x}, g_{0}(\mathbf{x})\right\rangle=0$, and we assume that $\mathbf{x} \in \Psi \Longleftrightarrow$ $\left\langle\mathbf{x}, g_{\mu}(\mathbf{x})\right\rangle=0$, with $g_{\mu}$ selfadjoint. By definition of confocality, $g_{\mu}^{-1}=g_{0}^{-1}-\mu$ id with $\mu \neq 0$. We are going to show below that

$$
\lambda g_{0}-\mu l_{\lambda} g_{\mu} l_{\lambda}-(\lambda-\mu) g_{\mu}=0 .
$$

$\mathbf{x} \in \Phi_{0} \cap \Psi$ together with (15) imply that $l_{\lambda}(\mathbf{x}) \in \Psi$, because

$$
\mu\left\langle l_{\lambda}(\mathbf{x}), g_{\mu} l_{\lambda}(\mathbf{x})\right\rangle=\mu\left\langle\mathbf{x}, l_{\lambda} g_{\mu} l_{\lambda}(\mathbf{x})\right\rangle=\lambda\left\langle\mathbf{x}, g_{0}(\mathbf{x})\right\rangle-(\lambda-\mu)\left\langle\mathbf{x}, g_{\mu}(\mathbf{x})\right\rangle=0 .
$$

We know (see the proof of Lemma 11) that $l_{\lambda}$ and $g_{0}$ commute, therefore so do $l_{\lambda}$ and $g_{\mu}^{-1}$. We verify (15) by multiplying its left hand side by $g_{\mu}^{-1}$ from the right:

$$
\begin{aligned}
& \lambda g_{0}\left(g_{0}^{-1}-\mu \mathrm{id}\right) \quad-\mu l_{\lambda} g_{\mu} l_{\lambda} g_{\mu}^{-1} \quad-(\lambda-\mu) \mathrm{id} \\
& =\lambda \mathrm{id}-\lambda \mu g_{0} \quad-\mu l_{\lambda} g_{\mu} g_{\mu}^{-1} l_{\lambda} \quad-(\lambda-\mu) \mathrm{id} \\
& =\lambda \mathrm{id}-\lambda \mu g_{0} \quad-\mu\left(\mathrm{id}-\lambda g_{0}\right)-(\lambda-\mu) \mathrm{id}=0 .
\end{aligned}
$$

Remark. There are up to $n$ confocal quadrics meeting in a non-absolute point of $\mathbb{P}^{n}$ (more are not possible because of their mutual orthogonality).

\subsection{Ivory's Theorem.}

Theorem 2. (Generalization of Ivory's Theorem) Assume that in a projective space $\mathbb{P}^{n}$ with metric (3) two regular quadrics $\Phi_{0}$ and $\Phi_{1}$ are confocal and of the same type. Then there is a smooth family $\Phi_{\lambda}=l_{\lambda}\left(\Phi_{0}\right)(0 \leq \lambda \leq 1)$ of quadrics confocal with $\Phi_{0}$ and $\Phi_{1}=l_{1}\left(\Phi_{0}\right)$, such that $l_{\lambda}$ is selfadjoint and has the Ivory property:

$$
\delta\left(\mathbf{x}, l_{\lambda}(\mathbf{y})\right)=\delta\left(l\left({ }_{\lambda} \mathbf{x}\right), \mathbf{y}\right) \text { for all } \mathbf{x}, \mathbf{y} \in \Phi_{0} .
$$

Any further quadric $\Psi$ confocal with $\Phi_{0}$ which contains a point $\mathbf{x} \in \Phi_{0}$ contains the entire path $l_{\lambda}(\mathbf{x})$, which intersects all quadrics $\Phi_{\lambda}$ orthogonally.

Proof: By Lemma 9, there exists $l$ such that $\Phi_{0}$ is defined by id $-l^{2}$ and $\Phi_{1}=l\left(\Phi_{0}\right)$. Lemma 11 shows the existence of $\Phi_{\lambda}$ and $l_{\lambda}$. By Lemma $6, l_{\lambda}$ has the 
Ivory property (16). At last, Lemma 12 shows the statement about the quadric $\Psi$, if it exists.

\section{REFERENCES}

[1] G. Albrecht: Eine Bemerkung zum Satz von Ivory. J. Geom. 50, 1-10 (1994).

[2] D. V. AlekseevskiJ, E. B. Vinberg and A. S. Solodovnikov: Geometry of spaces of constant curvature. in: Itogi Nauki i Tekhniki, Sovremennye Problemy Matematiki, Fundamentalnye Napravleniya, Vol. 29, VINITI, Moscow 1988; English translation in: Encyclopedia of Mathematical Sciences 29, Springer Verlag 1993.

[3] M. Berger: Geometry II. Springer-Verlag, Berlin Heidelberg 1987, p. 241.

[4] W. Blaschke: Analytische Geometrie. 3. Aufl., Verlag Birkhäuser, Basel 1954, p. 96.

[5] F. Dingeldey: Kegelschnitte und Kegelschnittsysteme. Encyklopädie der math. Wiss. III C 1, B.G. Teubner, Leipzig 1903. no. 65, p. 113.

[6] I. Gohberg, P. Lancaster and L. Rodman: Matrices and indefinite scalar products. Birkhäuser, Basel 1983.

[7] J. Ivory: On the Attractions of homogeneous Ellipsoids. Phil. Trans. of the Royal Society of London, 1809, 345-372.

[8] H. Stachel: Ivory's Theorem in the Minkowski Plane. Math. Pannonica 13, 11-22 (2002).

[9] H. Stachel: Configuration Theorems on Bipartite Frameworks. Rend. Circ. Mat. Palermo, II. Ser., 70, 335-351 (2002).

[10] H. Stachel: Flexible Octahedra in the Hyperbolic Space. Preprint: Institut für Geometrie, TU Wien, Technical Report 104 (2003).

[11] O. Staude: Flächen 2. Ordnung und ihre Systeme und Durchdringungskurven. Encyklopädie der math. Wiss. III C 2, B.G. Teubner, Leipzig 1904, no. 53, p. 204.

E-mail address: $\{$ stachel, wallner\}@geometrie.tuwien.ac.at

Institut für Geometrie, Technische Universität Wien, Wiedner Hauptstr. 8 10/113, A-1040 WIEN 